\title{
LA BIBLIOTECA Y LAS COMPETENCIAS INFORMACIONALES EN EL CURRÍCULO DE LOS ESTUDIANTES DE CIENCIAS, MEDICINA Y CIENCIAS DE LA SALUD DE LA UNIVERSIDAD DE ALCALÁ
}

\author{
The library and informational competences in the \\ curriculum of students of Sciences, Medicine, and \\ Health Sciences of the University of Alcalá, Spain
}

María-Isabel Domínguez-Aroca

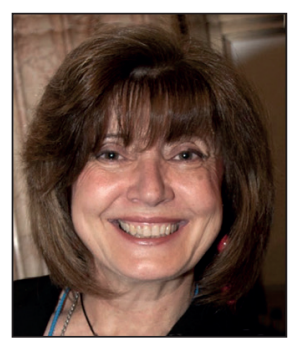

María-Isabel Domínguez-Aroca es diplomada en Formación del Profesorado de EGB por la Universitat de València (UV), licenciada en Historia por la Universidad de Alcalá (UAH) y documentalista especializada por la Universidad Complutense de Madrid (UCM). Es jefa de las bibliotecas del Área de Ciencias, Medicina y Ciencias de la Salud de la UAH. Ha formado parte del grupo Alfin/ Rebiun y ha sido representante de Rebiun en la sección de bibliotecas universitarias del Consejo de Cooperación Bibliotecaria del Ministerio de Cultura de 2007 a 2011, participando en el diseño y mantenimiento del portal www.alfared.org. Ha colaborado en encuentros de responsables de alfabetización en información organizados por Rebiun.

http://orcid.org/0000-0003-4237-1240

Universidad de Alcalá, Facultad de Medicina Ctra. Madrid-Barcelona, Km. 33,6 28871 Alcalá de Henares (Madrid), España misabel.dominguez@uah.es

\section{Resumen}

Se expone cómo las bibliotecas del Área de Ciencias, Medicina y Ciencias de la Salud de la Universidad de Alcalá (UAH) han implementado las competencias en información (CI) desde el curso 2010-2011 hasta el curso 2015-2016, principalmente de forma curricular, incluyéndolas en la mayoría de las guías docentes. Se indica la estrategia seguida, la información utilizada para lograr la colaboración con los docentes, la oportunidad que supuso el Espacio Europeo de Educación Superior (EEES), los antecedentes con los que se contaba, cómo ha sido la paulatina incorporación de las $\mathrm{Cl}$ en varios estudios y las indudables ventajas en el aprendizaje.

Palabras clave

Competencias informacionales; Alfin; Universidad de Alcalá; Bibliotecas universitarias; Educación superior; España.

\section{Abstract}

This paper aims to explain how the libraries in Sciences, Medicine, and Health Sciences at the University of Alcalá (UAH), Spain, have implemented information literacy (IL) in courses from 2010-2011 to 2015-2016, usually as part of the curriculum, including them in most teaching guides. This article discusses the strategy followed, the information available to achieve teacher collaboration, the opportunities offered by the European Higher Education Area (EHEA), the background data, the way IL has been gradually incorporated in various studies, and finally, the obvious advantages in the learning process.

\section{Keywords}

Information literacy; Information skills; University of Alcalá; Academic libraries; Higher education; Spain.

Domínguez-Aroca, María-Isabel (2017). “La biblioteca y las competencias informacionales en el currículo de los estudiantes de Ciencias, Medicina y Ciencias de la Salud de la Universidad de Alcalá". El profesional de la información, v. 26, n. 3, pp. 516-524.

https://doi.org/10.3145/epi.2017.may.18 


\section{Contexto para la formación en competencias en Ciencias, Medicina y Ciencias de la Salud de la Universidad de Alcalá}

El Área de Ciencias, Medicina y Ciencias de la Salud, situada en el campus externo de la Universidad de Alcalá, cuenta con cuatro bibliotecarios (incluyendo la responsable del Área) y tres bibliotecas para los estudios:

- Ciencias (Biología, Biología Sanitaria y Ciencias Ambientales);

- Farmacia (Farmacia y Química);

- Ciencias de la salud (Medicina, Enfermería, Fisioterapia, Ciencias de la Actividad Física y del Deporte).

Los bibliotecarios del Área tenían la idea clara de que la adquisición de competencias en información (Cl) es una materia necesaria en la formación universitaria (Área-Moreira, 2007), sabían que los caminos para conseguirlo pueden ser múltiples, y que todos tienen debilidades y fortalezas (Mahaffy, 2012; Tumblesson; Burke, 2010). La formación, parafraseando y adaptando los principios del curriculum attributes del proyecto Arcadia de la University of Cambridge (Secker; Coonan, 2011) pretende ser:

- holística: impregnando todo el proceso de estudio e investigación de los estudiantes, aunque se imparta en una asignatura, que sea aplicable a todas;

- modular: incluyéndola al menos cuando entran a la universidad en primero de grado y antes de salir de ella, apoyando la realización de su trabajo fin de grado TFG (o TFGM, trabajo fin de grado y máster, caso de Medicina y Farmacia);

- embebida: al integrarse en una asignatura del curriculum del alumno (siempre que ha sido posible) y con la presencia del profesor;

- activa y eminentemente práctica: tratando de trabajar con ejemplos reales en torno a los cuales se explican los recursos para acceder y gestionar la información, para que se sientan motivados e interesados;

- flexible: adaptándose a las características de cada titulación, organización interna, horarios de los estudios, etc.;

- transformadora: al conseguir que los alumnos aprecien y valoren la importancia y utilidad para sus estudios, para su trabajo futuro y para poder seguir aprendiendo por sí mismos el resto de su vida [Declaración de Alejandría (IFLA, 2005), Declaración de Toledo (MECD, 2006) (Lau, 2007)].

Los aliados fueron el profesorado, a pesar de que algunos estudios dicen que no siempre es fácil (Davidson, 2001) y el entusiasmo y responsabilidad de los bibliotecarios, que sabían que las $\mathrm{Cl}$ están vinculadas al éxito en los resultados en el aprendizaje (Virkus, 2003). Además, el curso académico 2008-2009 fue una oportunidad que podía aprovechar la biblioteca concebida como CRAI (centro de recursos para el aprendizaje y la investigación) (Domínguez-Aroca, 2005), pues empezaron algunos grados en el área y el EEES incorporaba las competencias en gestión de la información.

\section{Objetivo}

Este trabajo expone la experiencia llevada a cabo desde el curso 2010-2011 hasta el curso 2015-16, tiempo que se ha tardado en lograr incluir la formación en $\mathrm{Cl}$ en todas las titulaciones del área.
No se trata de describir ningún modelo ideal, sólo se muestra cómo es actualmente la implementación de las $\mathrm{Cl}$ y el recorrido llevado a cabo para hacerla realidad. Tampoco se pretende informar de forma exhaustiva de todos los cursos ofertados o impartidos, sino dar una visión global de los puntos clave y de las estratégicas seguidas.

\section{Estrategia y metodología}

La estrategia ha sido y sigue siendo "se hace camino al andar" como dice Machado, por ello los bibliotecarios elaboraron su propia pedagogía para paliar la falta de formación pedagógica, buscando los enfoques más efectivos en las diferentes situaciones formativas (Johnson; Blakesley, 2006), a pesar de las dificultades (Cull, 2005).

No suele ser conocido el papel que puede jugar la biblioteca en las $\mathrm{Cl}$ y se optó por tener un papel proactivo, informando de lo que hacían muchas bibliotecas universitarias españolas y extranjeras. La responsable de las bibliotecas del área, fue presentando y negociando alternativas con decanos o coordinadores de grado o profesores, ajustándose a las necesidades, realidades, tiempos, posibilidades y características de los estudios, siempre con el acuerdo previo de los bibliotecarios que realizaban principalmente la formación. Cada año resulta más sencillo y los argumentos se reducen a la evidencia, estableciéndose el calendario de forma consensuada. Las líneas clave seguidas:

- Transmitir en las negociaciones con el profesorado el marco de las $\mathrm{Cl}$ a nivel internacional, europeo, nacional, en la universidad y en la Universidad de Alcalá.

- Intentar dar la oportunidad a todos los estudiantes, de todas las titulaciones del área, para que adquiriesen las necesarias $\mathrm{Cl}$ al menos al entrar en la universidad y antes del TFG y cada vez más, en algunos de los masters que se imparten en el Área.

- Realizar una formación principalmente presencial (sin descartar los tutoriales de autoformación), con una media de dos horas, teniendo en cuenta el personal con el que se contaba.

- La formación debería estar consensuada con el profesor, tanto el nivel formativo como los temas de interés para la asignatura, los grupos, el aula, etc.

- La necesaria presencia del profesor en el aula, para que conociese lo que se enseñaba a sus alumnos, y lo qué podría exigirles, sin olvidar que normalmente se imparte en una hora que pertenece al profesor.

- Lograr que estuviese embebida en una asignatura siempre que fuese posible, o al menos darle el mayor nivel de obligatoriedad, e incluir la evidencia de la colaboración en las guías docentes.

- Cada año antes del verano, establecer el calendario de la formación curricular del curso siguiente, quedando así planificada, especialmente la del primer cuatrimestre.

- Hacer especial hincapié en los recursos de indudable calidad que la biblioteca adquiere y gestiona y pone a disposición de la comunidad universitaria, pues es evidente que lo que no se conoce no se utiliza. Con ello, la biblioteca puede contribuir a que la tasa de graduación y el éxito académico de los estudiantes sea superior entre aquellos que adquieren formación en $\mathrm{Cl}$, como dicen Oakleaf (2010) y Belanger et al. (2015), aunque 
"se dé la paradoja de que cuanto mayor es el esfuerzo por facilitar el acceso a la información digital, más invisibles se vuelven los servicios bibliotecarios a ojos de los usuarios" (Borrego, 2011).

Todo ello se realiza con la colaboración del profesorado, pues si éste se implica hay muchas más probabilidades de obtener éxito (Derakhshan; Singh, 2011).

\subsection{Competencias informacionales a nivel universi- tario en el contexto internacional, europeo y español}

Se informaba en las negociaciones que la integración de las $\mathrm{Cl}$ en los estudios superiores es una realidad en países como Estados Unidos y Australia (Virkus, 2003) estableciéndose en la política nacional de educación. En Europa, son múltiples los países que incorporan estas competencias en los planes docentes desde los primeros años de aprendizaje hasta postgrado, en Escocia y Gales (Open University Library Services, 2010), extendiéndose por Inglaterra y otros países europeos como Finlandia, considerada líder mundial en la elaboración de una política de $\mathrm{Cl}$. También la recogen organismos extranjeros e internacionales (ACRL (2016), Sconul, JISC, IFLA, Unesco, etc.), e incluso informes como el NMC Horizon report: Higher Education Edition 2016 (Johnson et al., 2016), o definiciones como las de Cilip (2013).

Un documento marco en Europa es:

- Recomendación del Parlamento Europeo al Consejo de 18 de diciembre de 2006 sobre competencias clave para el aprendizaje permanente (Unión Europea, 2006).

En España tenemos estos estudios:

- González-Fernández-Villavicencio, Domínguez-Aroca y Calderón-Rehecho (2013);

- Informe APEI (Calderón-Rehecho, 2010) que recoge algunas buenas prácticas de bibliotecas universitarias;

- monográfico de la Revista española de documentación científica de 2012 (REDC, 2012) sobre alfabetización informacional;

- plataforma Alfared del MECD y en concreto "Veintitantas experiencias alfin y una canción esperanzada" (MECD, 2011);

- Jornadas celebradas por Rebiun (Rebiun, 2009-2011);

- estudios recientes de Moreno-Pulido y Sánchez-Fernández (2015) y González-Fernández-Villavicencio et al. (2016).

- Competencias informáticas e informacionales (Cl2) en los estudios de grado, realizado por la Comisión Mixta CRUETIC (Rebiun, 2012).

- Real decreto 1393/2007, de 29 de octubre, modificado por el $R D$ 861/2010, por el que se establece la ordenación de las enseñanzas universitarias oficiales (España, 2007).

- Libros blancos de la Aneca ${ }^{1}$.

- Requisitos para la verificación de los títulos universitarios oficiales que habilitan para el ejercicio de profesiones reguladas (España, 2014), que recogen de una u otra forma la competencia de gestión de la información. Por ejemplo: - Farmacéutico

"Saber aplicar el método científico y adquirir habilidades en el manejo de la legislación, fuentes de información, bibliografía..." (España, 2008b).
- Médico:

"Ser capaz de formular hipótesis, recolectar y valorar de forma crítica la información para la resolución de problemas, siguiendo el método científico" (España, 2008a).

"Adquirir la formación básica para la actividad investigadora" (España, 2008a).

Se indica que el plan de estudios deberá incluir como mínimo, en el módulo de "Medicina Social, Habilidades de Comunicación e Iniciación a la Investigación":

"Conocer, valorar críticamente y saber utilizar las tecnologías y fuentes de información clínica y biomédica, para obtener, organizar, interpretar y comunicar información clínica, científica y sanitaria [...] Manejar con autonomía un ordenador personal. Usar los sistemas de búsqueda y recuperación de la información biomédica" (España, 2008a).

Rebiun plantea la necesidad de adaptar el decálogo $\mathrm{Cl} 2$ y su desarrollo competencial al proyecto DigComp ${ }^{2}$ de la Comisión Europea (Ferrari, 2013), como propuesta de marco común de referencia de competencias digitales en Europa, en el cual la primera de las competencias digitales es la información.

\subsection{Competencias informacionales en la Universidad de Alcalá}

Podríamos resaltar:

a) En el modelo educativo (UAH, 2007) se incluyen las tecnologías de la información y la comunicación. Entre las competencias procedimentales:

- gestión de la información y del conocimiento;

- uso de las tecnologías de la información y la comunicación.

b) La oferta de titulaciones de la $U A H$, que en todas las ramas de enseñanza incluye la competencia en gestión de la información de distintas formas. Ejemplo:

- Farmacia, competencia no 3:

"Saber aplicar el método científico y adquirir habilidades en el manejo de legislación, fuentes de información, bibliografía".

http://farmacia.uah.es/estudios/grado-int.asp?cd=104\&plan=G57

- Medicina, competencia n. 12:

"Conocer, valorar críticamente y saber utilizar las fuentes de información clínica y biomédica para obtener, organizar, interpretar y comunicar la información científica y sanitaria".

http://medicinaycienciasdelasalud.uah.es/estudios/gradoint. asp?cd $=101$ \& plan $=$ G215

c) La normativa reguladora de la oferta de asignaturas y actividades transversales en las titulaciones de grado:

“b) Desarrollar competencias transversales.... capacitación en el uso de las TIC; Iniciación a la Investigación; cultura profesional, búsqueda y gestión de la información..." (UAH, 2009).

d) El Plan de Formación de usuarios existente en la Biblioteca de la Universidad de Alcalá.

https://biblioteca.uah.es/biblioteca/documentos/plan_ formacion_2013-15.pdf 
Todo lo expuesto, sumado a las preocupaciones de los docentes sobre el uso indiscriminado y generalizado de internet por los alumnos, la preocupación por evitar el plagio y el ciberplagio, por ser una de las prácticas deshonestas más usuales del universitario (Domínguez-Aroca, 2012), (Comas-Forgas et al., 2011) frente a la escasez de uso de las fuentes de calidad que proporciona la Biblioteca para recuperar información (Serra-Ferrer, 2015) denotaba la falta de $\mathrm{Cl}$. Todos estos hábitos han sido recogidos por numerosos estudios entre los que podíamos citar los de OCLC, 2002; LibQUAL+TM (Cook et al., 2004, 2007); Marzal y CalzadaPrado (2004); Griffiths y Brophy (2005); Sureda-Negre y Comas-Forgas (2006).

\section{Implementación de las $\mathrm{Cl}$ curriculares}

En el contexto descrito, docentes y bibliotecarios comenzaron a trabajar juntos con el objetivo común de mejorar las $\mathrm{Cl}$ de los alumnos en su paso por la universidad.

Las bibliotecas del área tenían experiencia en formación individual y con grupos reducidos por propia iniciativa. Esto suponía resultados muy positivos para ambas partes, quien recibe y quien forma. Por ejemplo, en los estudios de Ciencias de la Actividad Física y del Deporte (Ccafyde) se impartía formación de la base de datos Sportdiscus en una asignatura; o la compra de la base de datos SciFinder en 2006 que supuso la organización de cursos de formación dirigidos principalmente a profesores y a alumnos de licenciatura.

La incorporación ha sido progresiva en los estudios del Área y de forma principalmente curricular. En la colaboración queda establecida:

- Reunión previa de los bibliotecarios con el docente: para conocer las materias de interés de la asignatura y el nivel de conocimientos que el docente considera deben adquirir sus alumnos.

- Duración: se optó por impartir dos horas de formación pues al hacerla extensiva a las nueve titulaciones era la opción más realista y posible, no hay que olvidar que es una de las muchas tareas que se realizan en la biblioteca.

- Fecha: la mayor concentración de la formación es al comienzo del curso para los nuevos alumnos de grado, en los meses de septiembre y octubre, aunque se sigue impartiendo a lo largo del curso.

- División en grupos: se establecen los grupos, el número de sesiones que recibirá el alumno y el nivel formativo.

- Lugar: generalmente en el aula para los alumnos del primer curso de grado (excepto Ccafyde, Ambientales y Enfermería que se realiza en el aula de informática en grupos) y el resto (2으. $3 \circ$, TFG, etc.) divididos en grupos, se imparte en un aula de informática.

- Presencia del profesor: la necesaria presencia del profesor durante la formación (suele ser lo habitual).

- Evaluación de la formación: necesidad de elaborar o no tests de preguntas, prácticas o cualquier otra forma que evidencie que han adquirido la formación impartida.

- Se acuerda el envío al profesor del material utilizado.

- Cuestionario de evaluación: En los casos en los que la formación se imparte en un aula de informática mediante de un cuestionario de evaluación online.

https://biblioteca.uah.es/servicios/CuestionarioEvaluacion.asp

- Materiales de autoaprendizaje: se informa de los tutoriales que tiene la Biblioteca para el desarrollo de las $\mathrm{Cl}$.

De forma resumida la implementación de la formación por titulaciones se muestra en las tablas 1 a 8.

Tabla 1. Biología y biología sanitaria

\begin{tabular}{|c|c|c|c|c|}
\hline Curso & Asignatura & Modalidad & Grupos & Horas \\
\hline En $1^{\circ}$ de grado. Desde el curso 2014-15 & $\begin{array}{l}\text { "Métodos en biodiversidad y conservación". En Biología } \\
\text { sanitaria en "Biología celular e histología" }\end{array}$ & Presencial & 2 & 2 \\
\hline En $4^{\circ}$ de grado, en el TFG. Desde el curso 2015-16 & $\begin{array}{l}\text { Seminario específico coordinado por el Decanato, no em- } \\
\text { bebido en ninguna asignatura }\end{array}$ & Presencial & 2 & 2 \\
\hline
\end{tabular}

Tabla 2. Ciencias de la actividad física y el deporte

\begin{tabular}{|c|c|c|c|c|}
\hline Curso & Asignatura & Modalidad & Grupos & Horas \\
\hline $\begin{array}{l}\text { En } 2^{\circ} \text { de grado. } \\
\text { Curso } 2009-10\end{array}$ & $\begin{array}{l}\text { "Sociología del deporte. Metodología del trabajo } \\
\text { científico" }\end{array}$ & Semipresencial & 2 & $\begin{array}{l}6 \text { sesiones } \\
\text { de } 45 \\
\text { minutos }\end{array}$ \\
\hline $\begin{array}{l}\text { Curso 2010-11 a 2013-14, se impartía en todos } \\
\text { los grados. } \\
\text { Se creó un grupo de innovación }{ }^{3}\end{array}$ & Comenzó en "Historia del Deporte" & Presencial & 2 & 2 \\
\hline $\begin{array}{l}\text { En } 1^{\circ} \text { de Grado. Desde el curso 2014-15 } \\
\text { (esta formación sustituyó la anterior) }\end{array}$ & "Fundamentos de atletismo" & Presencial & 2 & 2 \\
\hline $\begin{array}{l}\text { En } 3^{\circ} \text { de grado. } \\
\text { Desde el curso } 2014-15\end{array}$ & $\begin{array}{l}\text { "Iniciación a la investigación en Ciencias de la activi- } \\
\text { dad física y del deporte" }\end{array}$ & Presencial & 2 & 2 \\
\hline En $4^{\circ}$ de grado desde $2014-15$ para el TFG & $\begin{array}{l}\text { Seguimiento individual o en pequeños grupos en la } \\
\text { biblioteca }\end{array}$ & Presencial & & Variables \\
\hline
\end{tabular}

Evidencia en la mayoría de las guías docentes de la titulación.

https://www.uah.es/es/estudios/estudios-oficiales/grados/asignaturas/index.htm/?codCentro=101\&codPlan=G770 
Tabla 3. Ciencias ambientales

\begin{tabular}{|c|c|c|c|c|}
\hline Curso & Asignatura & Modalidad & Grupos & Horas \\
\hline En $1^{\circ}$ de grado. Desde el curso 2011-12 & "Bioquímica" & Presencial & 2 & 2 \\
\hline En $3^{\circ}$ de grado. Desde el curso 2012-13 & $\begin{array}{l}\text { "Hidrología e hidrogeología" y "Ordenación del territorio, medio } \\
\text { físico y humano" (dos profesoras se ponen de acuerdo) }\end{array}$ & Presencial & 2 & 2 \\
\hline
\end{tabular}

Evidencia: en las guías docentes de la asignatura de 1 으 de grado han añadido tutoriales de la Biblioteca en la bibliografía. https://www.uah.es/es/estudios/estudios-oficiales/grados/asignaturas/index.htm/?codCentro=107\&codPlan=G670 https://biblioteca.uah.es/servicios/formacion.asp?capa=autoformacion

Tabla 4. Enfermería

\begin{tabular}{|l|l|l|l|}
\hline \multicolumn{1}{|c|}{ Curso } & \multicolumn{1}{|c|}{ Asignatura } & \multicolumn{1}{|c|}{ Modalidad } \\
\hline $\begin{array}{l}\text { En } 1^{\circ} \text { de grado. Desde el curso } \\
2009-10\end{array}$ & $\begin{array}{l}\text { "Búsqueda y gestión de información en ciencias } \\
\text { sociosanitarias" }\end{array}$ & $\begin{array}{l}\text { Presencial } \\
\text { (la biblioteca envía los ejer- } \\
\text { cicios de evaluación para el } \\
\text { aula virtual) }\end{array}$ & $\begin{array}{c}\text { G sesiones de 2 h. } \\
\text { Cada alumno } \\
\text { recibe 4:30 h. }\end{array}$ \\
\hline $\begin{array}{l}\text { En } 4^{\circ} \text { de grado desde el curso } \\
2015-16\end{array}$ & Asignatura del TFG & Presencial \\
\hline
\end{tabular}

En la guía docente de 1ㅇ de grado se incluye la participación de la Biblioteca, introduciendo en el apartado del profesorado, colaboradores, donde se indican los bibliotecarios que participan en la formación y la biblioteca a la que pertenecen.

https://www.uah.es/export/sites/uah/es/estudios/estudios-oficiales/grados/.galleries/Programas/G61/100003_G61_2016-17.pdf

Tabla 5. Farmacia

\begin{tabular}{|c|c|c|c|c|}
\hline Curso & Asignatura & Modalidad & Grupos & Horas \\
\hline En $1^{\circ}$ de grado. Curso $2010-11$ & "Historia de la farmacia y metodología científica" & Presencial & 2 & $\begin{array}{l}1 \text { h. (comenzó } \\
\text { con } 2 \text { h.) }\end{array}$ \\
\hline En $5^{\circ}$ de grado. Curso 2014-15 & $\begin{array}{l}\text { Optativa: "Iniciación a la investigación en ciencias farma- } \\
\text { céuticas" }\end{array}$ & Presencial & 1 & 4 \\
\hline
\end{tabular}

En la guía docente de 1을 grado.

https://www.uah.es/export/sites/uah/es/estudios/estudios-oficiales/grados/.galleries/Programas/G57/570006_G57_2016-17.pdf

En la guía docente de 5ㅇ de grado de Farmacia se incorpora en el apartado de "Contenidos", en la parte de "Prácticos" donde se enumeran los temas que imparte la biblioteca.

https://www.uah.es/export/sites/uah/es/estudios/estudios-oficiales/grados/.galleries/Programas/G57/570039_G57_2016-17.pdf

Tabla 6. Fisioterapia

\begin{tabular}{|c|c|c|c|c|}
\hline Curso & Asignatura & Modalidad & Grupos & Horas \\
\hline $\begin{array}{l}\text { En } 1^{\circ} \text { de grado, desde el curso 2016-2017 } \\
\text { (comenzará este curso) }\end{array}$ & $\begin{array}{l}\text { "Bases teóricas de la aplicación de los agentes físicos en } \\
\text { Fisioterapia" }\end{array}$ & Presencial & 2 & 2 \\
\hline En $4^{\circ}$ de grado desde el curso $2012-2013$ & TFG & Presencial & 3 & $2: 30$ \\
\hline
\end{tabular}

En la guía docente del TFG de 4ㅇ de grado, la colaboración de la biblioteca aparece en el apartado de "Estrategias metodológicas".

https://www.uah.es/export/sites/uah/es/estudios/estudios-oficiales/grados/.galleries/Programas/G249/249022_G249_2016-17.pdf

Tabla 7. Medicina

\begin{tabular}{|c|c|c|c|c|}
\hline Curso & Asignatura & Modalidad & Grupos & Horas \\
\hline $\begin{array}{l}\text { En } 1^{\circ} \text { de grado. Desde el curso } \\
2011-12\end{array}$ & "Biología: citología y citogenética médicas" & Presencial & 1 & 2 \\
\hline \multirow{2}{*}{$\begin{array}{l}\text { En } 2^{\circ} \text { de grado. Desde el curso } \\
2011-12\end{array}$} & \multirow{2}{*}{$\begin{array}{l}\text { "Iniciación a la investigación, documentación científica y TICs" } \\
\text { La Biblioteca es responsable de las prácticas, son obligatorias y } \\
\text { suponen el } 20 \% \text { de la calificación. }\end{array}$} & \multirow{2}{*}{ Semipresencial } & 1 & 2 \\
\hline & & & 4 & $\begin{array}{l}6 \mathrm{~h} \text {. presenciales } \\
\text { y } 11 \text { virtuales }\end{array}$ \\
\hline
\end{tabular}

En la guía docente de 2ㅇ de grado aparece la colaboración de la Biblioteca y se incluyen sus tutoriales en la bibliografía.

http://www.uah.es/export/sites/uah/es/estudios/estudios-oficiales/grados/.galleries/Programas/G215/215013_G215_2016-17.pdf

Se presentó un poster en el Encuentro BibioSalud en 2014, donde se recogían los resultados de la participación de tres cursos académicos de los alumnos (Domínguez-Aroca; Iglesia-Sánchez, 2014). 
Tabla 8. Química

\begin{tabular}{|c|c|c|c|c|}
\hline Curso & Asignatura & Modalidad & Grupos & Horas \\
\hline En $1^{\circ}$ de grado. Desde el curso 2014-15 & "Biología" & Presencial & 1 & 2 \\
\hline En $2^{\circ}$ de grado. Curso 2013-14 & "Química analítica I y II" & Presencial & 2 & 2 \\
\hline En $3^{\circ}$ de grado. Desde el curso 2015-16 & "Introducción a la síntesis orgánica" & Presencial & 3 & 2 \\
\hline
\end{tabular}

Aparece la colaboración de la biblioteca en las guías docentes de 1으 y de 3으 de grado.

https://www.uah.es/es/estudios/estudios-oficiales/grados/asignaturas/index.htm/?codCentro=110\&codPlan=G660

Observación: Los enlaces a las guías docentes se actualizan cada curso académico, por lo que será necesario entrar cada año en la correspondiente guía docente de la asignatura para consultarla.

Podríamos resaltar especialmente la experiencia con la titulación de Ccafyde, la de Medicina y la de Enfermería. En la primera porque se asiste a las reuniones de grado y un grupo numeroso de profesores asume el compromiso de implementar las $\mathrm{Cl}$ de forma coordinada; Medicina, porque la biblioteca se encarga de las prácticas en la asignatura "Iniciación a la investigación, documentación científica y TICs" y en Enfermería porque fue abriendo las puertas de las demás.

A modo de resumen se incorporan dos gráficos que muestra la formación impartida en el período 2011-15 en el Área, tanto a los alumnos como al personal docente e investigador (PDI), curricular y no curricular (figura 1). La figura 2, indica el número de horas utilizadas por los bibliotecarios y el número de cursos impartidos. Los datos que se muestran se refieren a años naturales (según se proporcionan en las estadísticas anuales a Rebiun).

https://rebiun.um.es/rebiun/admin/ManagelndicatorsPage

\section{Resultados y conclusiones}

La colaboración y participación de los bibliotecarios en el aprendizaje han sido beneficiosas para los alumnos y para las relaciones entre el bibliotecario y el docente ya que "está pasando por uno de sus mejores momentos" (González-Fernández-Villavicencio et al., 2016).

Actualmente el profesor ya no es la fuente única del saber, pues comparte competencias con textos, especialistas, expertos, compañeros, personas de otras culturas, bases documentales, etc. (De-Pablos-Pons, 2010). Pongamos nuestro granito de arena los bibliotecarios y trabajemos desde una concepción de "aprendizaje expansivo" (Iborra; Domínguez-Aroca, 2013).

La formación está teniendo indudables ventajas, los alumnos consiguen realizar trabajos de mayor calidad, aprenden a evaluar la información que utilizan y son más éticos en el uso de la misma; incluso los profesores son más exigentes a la hora de evaluar la información que utilizan sus alumnos y cómo la referencian. Contamos con algunas evidencias propias que lo corroboran: las evaluaciones realizadas, los resultados expuestos en las XV Jornadas nacionales de información y documentación en ciencias de la salud (Domínguez-Aroca; Iglesia-Sánchez, 2014) que recogía las opiniones del profesorado implicado, o las del $V$ Encuentro sobre innovación en docencia universitaria (Arévalo-Baeza; Domínguez-Aroca; Iglesia-Sánchez, 2012).

Se ha conseguido que el profesorado reconozca que los bibliotecarios son los más adecuados para impartir las $\mathrm{Cl}$, pues éstos han tenido que adaptarse a las nuevas formas en las que se presenta y se accede a la información, habilidades que en el entorno actual de cambios tecnológicos constantes, diversidad y cantidad de información, lo sitúan en una posición ventajosa frente a alumnos, docentes e investigadores.

Este trabajo tiene entre sus limitaciones el que la formación no esté embebida al mismo nivel en todas las titulaciones, ni cuente con la misma implicación y convencimiento de todos los profesores, pero se trabaja cada día para que la valoren y exijan a sus alumnos como parte de las competencias a desarrollar en el curriculum. Es un camino a veces difícil y lento, pero es necesario ser constante, mejorar e innovar para adaptarse a las nuevas necesidades y realidades de los usuarios que cada año entran en la Universidad y necesitan recibir formación en $\mathrm{Cl}$.

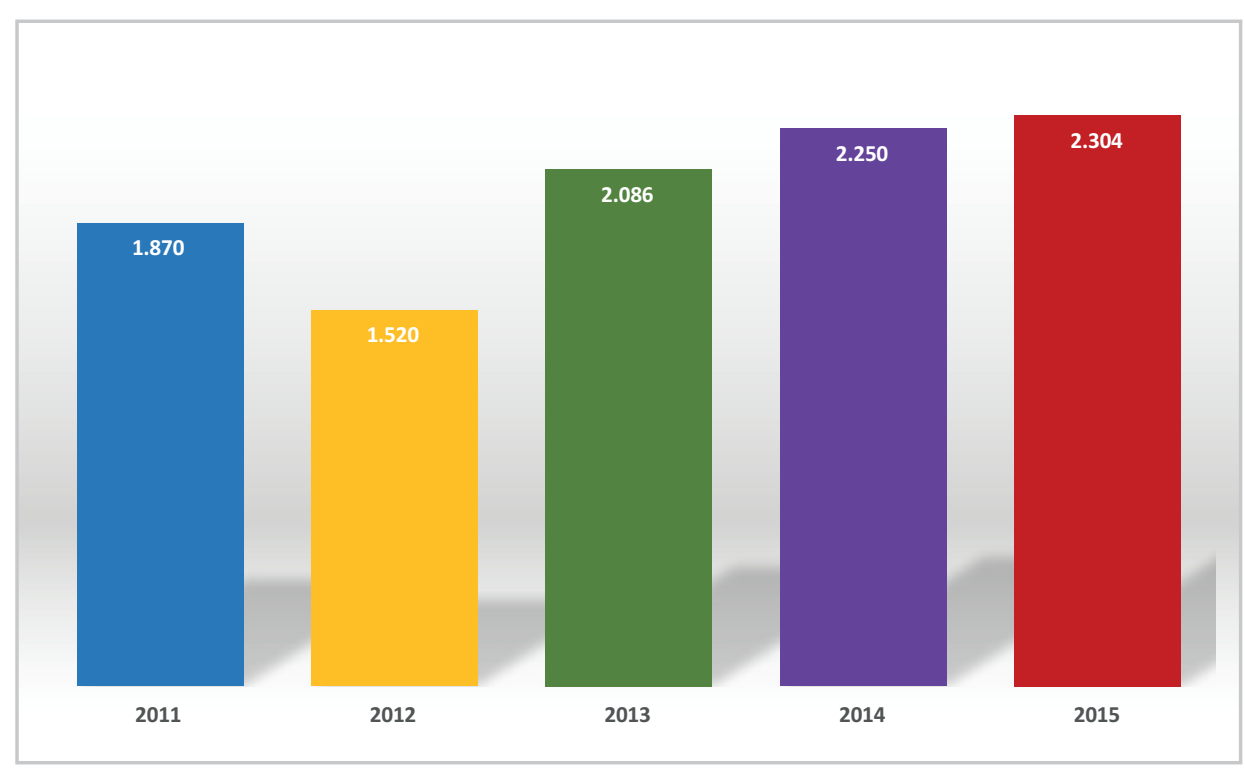

Figura 1. Número de asistentes a la formación en competencias en información (CI) en el período 2011-2015. 


\section{Agradecimientos}

Agradecer con este testimonio el esfuerzo continuado de mis compañeras por su trabajo diario durante años para mejorar las competencias informacionales de alumnos y profesores y la inestimable colaboración pasada, presente y esperemos que futura de los profesores.

\section{Notas}

1. Libros blancos. Elaborados por una red de universidades españolas, apoyadas por la Aneca, cuyo objetivo era realizar estudios y supuestos prácticos útiles en el diseño

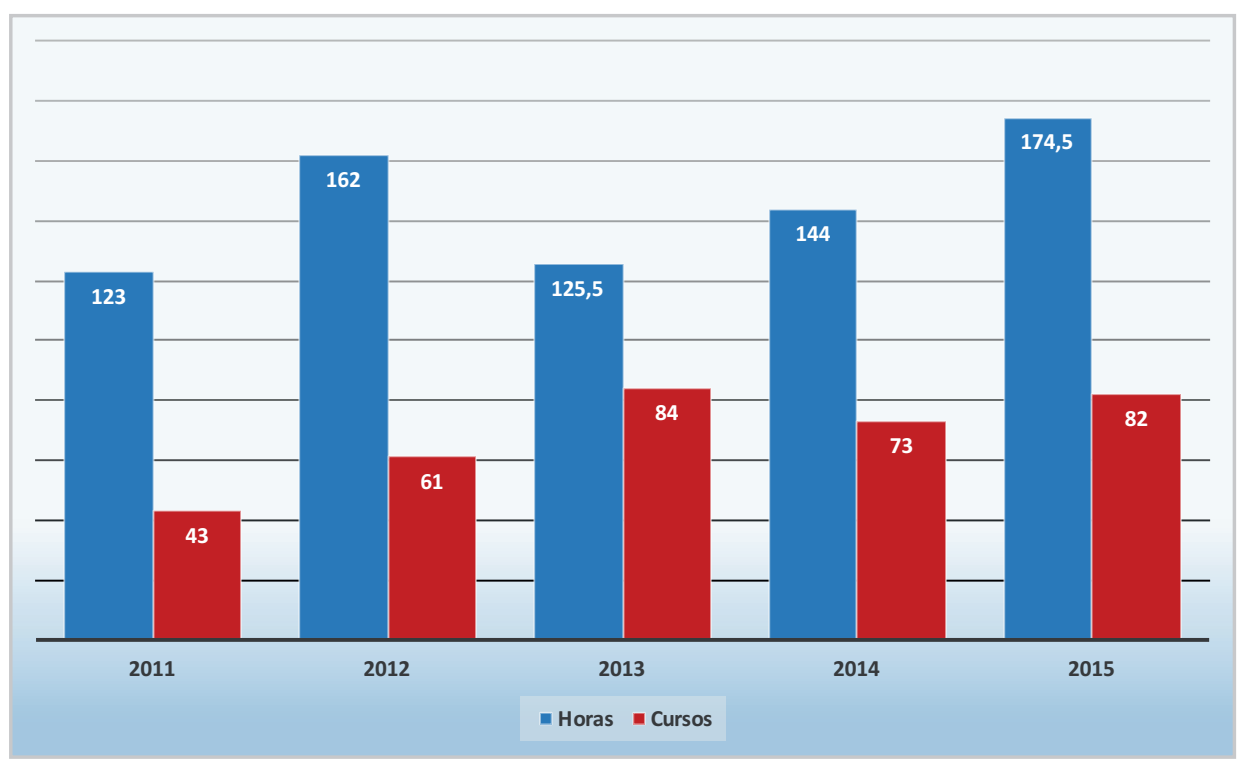

Figura 2. Número de horas por año y número de cursos en el período 2011-2015. de títulos de grado adaptados al EEES.

http://www.aneca.es/Documentos-y-publicaciones/Otrosdocumentos-de-interes/Libros-Blancos

2. Digcomp plantea un nuevo paradigma en Rebiun al introducir nuevos conceptos: competencia digital, alfabetización digital, e-habilidades, alfabetización mediática, lo que implica revisar la formación anterior basada en competencias informáticas e informaciones:

http://www.rebiun.org/competenciadigital/Paginas/default. aspx

3. Grupo de Innovación Educativa denominado: Reconstruir la práctica a través del desarrollo interdisciplinar y el trabajo colaborativo en el Grado de ciencias de la actividad física y del deporte y con número de registro: UAH-GI10-21.

\section{Bibliografía}

ACRL (2016). Framework for information literacy for higher education. Filed by the ACRL Board on February 2, 2015. Adopted by the ACRL Board, January 11, 2016. ACRL (Association of College and Research Libraries).

http://www.ala.org/acrl/sites/ala.org.acrl/files/content/ issues/infolit/Framework_ILHE.pdf

Área-Moreira, Manuel (2007). Documento marco de Rebiun para la CRUE: adquisición de competencias en información, una materia necesaria en la formación universitaria. La Laguna. http://www.rebiun.org/documentos/Documents/IIPE_ LINEA1_07-11/IIPE_Linea1_Adquisicionencompetencias_ Resumen_2007.pdf

Arévalo-Baeza, Marta; Domínguez-Aroca, María-Isabel; Iglesia-Sánchez, Amparo (2012). "Trabajo colaborativo e interdisciplinar para el desarrollo de competencias transversales informacionales: una experiencia en el Grado de Ciencias de la actividad física y del deporte". En: García-Campos, María-Dolores; Canabal-García, Cristina (dir.); Viejo-Díaz, Carmen (coord.). Aproximaciones a la coordinación docente: hacia el cambio en la cultura universitaria. 5o Encuentro sobre innovación en docencia universitaria. Universidad de Alcalá, 15 de marzo de 2011, pp. 61-83. ISBN: 978

8481389524

http://eprints.rclis.org/15565

Belanger, Jackie; Zou, Ning; Mills, Jenny-Rushing; Holmes, Claire; Oakleaf, Megan (2015). "Project Rails: Lessons learned about rubric assessment of information literacy skills". Portal: Libraries and the Academy, v. 15, n 4, pp. 623-644.

https://www.press.jhu.edu/journals/portal_libraries_and_ the_academy/portal_pre_print/articles/belanger.pdf

Borrego, Ángel (2011). "El valor de la biblioteca para la investigación”. Blok de bid, 20 abril.

http://www.ub.edu/blokdebid/es/node/89

Calderón-Rehecho, Andoni (2010). Informe APEI sobre alfabetización informacional. Asociación profesional de especialistas en información, Ministerio de Cultura. http://eprints.rclis.org/14972

Cilip (2013). "Information literacy - Definition". Cilip. The library and information association.

http://eprints.rclis.org/7459/1/Article_Update_25102004.pdf

Comas-Forgas, Rubén; Sureda-Negre, Jaume; Casero, Antonio; Morey, Mercè (2011). "La integridad académica entre el alumnado universitario español”. Estudios pedagógicos (Valdivia), v. 37, n. 1, pp. 225-225.

http://www.scielo.cl/scielo.php?script=sci_arttext\&pid =S0718-07052011000100011

Cook, Colleen; Heath, Fred; Thompson, Bruce; Askew, Consuella; Kyrillidou, Martha; Webster, Duane (2004). LibQUAL+TM spring 2004 survey. Washington, DC: Association of Research Libraries (ARL).

http://www.libqual.org/documents/admin/ARL_notebook2004. $p d f$

Cook, Colleen; Heath, Fred; Thompson, Bruce; Davis, MaShana; Kyrillidou, Martha; Webster, Duane (2007). LibQUAL+TM spring 2007 survey. Washington, DC.: Association of Researcher Libraries (ARL).

http://www.libqual.org/documents/admin/ARL_Notebook. $p d f$ 
Cull, Barry W. (2005). "Voices in the wilderness: A report on academic information literacy instruction in Atlantic Canada". Canadian journal of information and library science, $v$. 29 n. 1, pp. 1-26.

https://lib.unb.ca/instruction/bcull/CJILS2005.pdf

Davidson, Jeanne R. (2001). "Faculty and student attitudes toward credit courses for library skills". College and research libraries, v. 62, n. 2, pp. 155-163.

http://pdxscholar.library.pdx.edu/cgi/viewcontent. cgi? article $=1138 \&$ context $=u l i b \_f a c$

Derakhshan, Maryam; Singh, Diljit (2011). "Integration of information literacy into the curriculum: A meta-synthesis". Library review, v. 60, n. 3, pp. 218-229.

https://goo.gl/K1XosA

http://dx.doi.org/10.1108/00242531111117272

Domínguez-Aroca, María-Isabel (2005). “La biblioteca universitaria ante el nuevo modelo de aprendizaje: docentes y bibliotecarios, aprendamos juntos porque trabajamos juntos". RED. Revista de educación a distancia, n. monográfico IV, julio.

http://revistas.um.es/red/article/view/24481/23821

Domínguez-Aroca, María-Isabel (2012). “Lucha contra el plagio desde las bibliotecas universitarias". El profesional de la información, v. 21, n. 5, pp. 498-503.

https://doi.org/10.3145/epi.2012.sep.08

Domínguez-Aroca, María-Isabel; Iglesia-Sánchez, Amparo (2014). "Competencias en información en el Grado en Medicina de la Universidad de Alcalá: una experiencia de participación" [poster]. En: 15a Jornadas nacionales de información y documentación en ciencias de la salud. Madrid, 22-23 de mayo.

https://www.pinterest.com/pin/435441857694249033

España (2007). "Real decreto 1393/2007, de 29 de octubre, por el que se establece la ordenación de las enseñanzas universitarias oficiales". Boletín oficial del Estado, n. 260, 30 de diciembre.

https://www.boe.es/buscar/act.php?id=BOE-A-2007-18770

España (2008a). “Orden ECl/332/2008, de 13 de febrero, por la que se establecen los requisitos para la verificación de los títulos universitarios oficiales que habiliten para el ejercicio de la profesión de médico". Boletín oficial del Estado, n. 40, 15 de febrero.

http://www.boe.es/buscar/doc.php?id=BOE-A-2008-2674

España (2008b). “Orden CIN/2137/2998, de 3 de julio, por la que se establecen los requisitos para la verificación de los títulos oficiales que habiliten para el ejercicio de la profesión de farmacéutico". Boletín oficial del Estado, n. 174, 19 de julio.

http://www.boe.es/diario_boe/txt.php?id=BOE-A-2008-12391

España (2014). "Real decreto 967/2014, de 21 de noviembre, por el que se establecen los requisitos y el procedimiento para la homologación y declaración de equivalencia a titulación y a nivel académico universitario oficial y para la convalidación de estudios extranjeros de educación superior, y el procedimiento para determinar la correspondencia a los niveles del marco español de cualificaciones para la educación superior de los títulos oficiales de arquitecto, ingeniero, licenciado, arquitecto técnico, ingeniero técnico y diplomado". Boletín oficial del Estado, n. 283, 22 de noviembre.

https://www.boe.es/buscar/act.php?id=BOE-A-2014-1209

Ferrari, Anusca (2013). Digcomp: A framework for developing and understanding digital competence in Europe. Luxembourg: Publications Office of the European Union. Report EUR 26035EN. ISBN: 9789279314650

http://ftp.jrc.es/EURdoc/JRC83167.pdf

González-Fernández-Villavicencio, Nieves; Barrera-Gómez, Juan-Antonio; Saen-De-Casas, Eloisa; Moya-Orozco, Víctor M. (2016). Bibliotecarios e innovación docente: La oportunidad de los planes tutoriales y los trabajos de fin de grado. Bid, n. 36.

https://doi.org/10.1344/BiD2016.36.14

González-Fernández-Villavicencio, Nieves; DomínguezAroca, María-Isabel; Calderón-Rehecho, Andoni (2013). "State of the art of information literacy in Spanish university libraries and a proposal for the future". En: Worldwide commonalities and challenges in information literacy research and practice. $1^{\text {st }}$ European conf on information literacy, ECIL 2013; Istanbul, Turkey; 22-25 October. Switzerland: Springer International Publishing, v. 397, pp. 288-294. ISBN: 978 3319039180

https://goo.gl/eRLiOt

https://doi.org/10.1007/978-3-319-03919-0_37

Griffiths, Jillian R.; Brophy, Peter (2005). "Student searching behavior and the web: use of academic resources and Google". Library trends, v. 53, n. 4, pp. 539-554.

http://citeseerx.ist.psu.edu/viewdoc/download?doi=10.1.1. 172.8640\& $\mathrm{rep}=$ rep $1 \&$ type $=p d f$

Iborra, Alejandro; Domínguez-Aroca, María-Isabel (2013). "Investigador y bibliotecario: un ejemplo de colaboración expandida". En: Seminario Madroño. I Encuentro de investigadores y bibliotecarios: alianzas y expectativas, 27 de nov. http://www.consorciomadrono.es/noticias_eventos/2013/ invesmasbiblio13/UAH.pdf

IFLA (2005). Faros para la sociedad de la información. Declaración de Alejandría acerca de la alfabetización informacional y el aprendizaje de por vida. IFLA.

https://www.ifla.org/node/7275

Johnson, Corey M.; Blakesley, Elisabeth (2006). "Why we do what we do: Exploring priorities within public services librarianship". Portal: Libraries and the academy, v. 6, n. 3, pp. 347-369.

https://doi.org/10.1353/pla.2006.0040

Johnson, Larry; Adams-Becker, Samantha; Cummins, Michele; Estrada, Victoria; Freeman, Alex; Hall, Courtney (2016). NMC Horizon report. Edición educación superior 2016. Austin, Texas: The New Media Consortium.

http://research.unir.net/wp-content/uploads/2016/05/2016nmc-horizon-report-HE-ES.pdf

Lau, Jesús (2007). Directrices sobre desarrollo de habilidades informativas para el aprendizaje permanente. La Haya: IFLA. 
http://www.ifla.org/files/assets/information-literacy/ publications/ifla-guidelines-es.pdf

Mahaffy, Mardi (2012). "Student use of library research guides following library instruction". Communications in information literacy, v. 6, n. 2, pp. 202-213.

https://goo.gl/c2ZoXI

Marzal, Miguel-Ángel; Calzada-Prado, Javier (2003). “Un análisis de necesidades y hábitos informativos de estudiantes universitarios en internet". Binaria: Revista de comunicación, cultura y tecnología, n. 3.

http://e-archivo.uc3m.es/handle/10016/4632

Ministerio de Educación, Cultura y Deporte (2006). "Declaración de Toledo. Bibliotecas por el aprendizaje permanente". En: Biblioteca, aprendizaje y ciudadanía: alfabetización informacional. Toledo, Biblioteca Regional de Castilla-La Mancha, 2-3 de febrero.

http://travesia.mcu.es/portalnb/jspui/bitstream/10421/1545/1/ Alfin.pdf

Ministerio de Educación, Cultura y Deporte (2011). "Veintitantas experiencias alfin y una canción esperanzada". Foro RED alfabetización informacional. Ministerio de Educación, Cultura y Deporte; Secretaría de Estado de Cultura.

http://www.alfared.org/content/veintitantas-experienciasalfin-y-una-canci-n-esperanzada/1287

Moreno-Pulido, Alexis; Sánchez-Fernández, Rosa (2015). "Papel de la biblioteca en la enseñanza de competencias informacionales en posgrados de la Universidad Nacional de Educación a Distancia (UNED)". El profesional de la información, v. 24, n. 5, pp. 665-673.

http://dx.doi.org/10.3145/epi.2015.sep.15

Oakleaf, Megan (2010). The value of academic libraries: $A$ comprehensive research review and report. Chicago: Association of College \& Research Libraries. ISBN: 9780838985687 http://www.ala.org/acrl/sites/ala.org.acrl/files/content/ issues/value/val_report.pdf

OCLC (2002). "How academic librarians can influence students' web based information choices". OCLC White paper on the information habits of college students. Ohio: OCLC, June. http://www.aect.org/publications/whitepapers/2010/ informationhabits.pdf

Open University Library Services (2010). Integrating information literacy into the curriculum. Open University Library Services. COBE (Centre for Outcomes-Based Education). The Open University.

http://www.open.ac.uk/libraryservices/documents/ Integrating_IL_Booklet_2010.pdf

De-Pablos-Pons, Juan-Manuel (2010). “Universidad y sociedad del conocimiento. Las competencias informacionales y digitales". RUSC. Universities and knowledge society journal, v. 7, n. 2.

https://dialnet.unirioja.es/descarga/articulo/3666613.pdf

Rebiun (2009-2011). Jornadas de alfabetización informacio- nal (alfin). Jornadas de trabajo de responsables de alfabetización informacional en bibliotecas universitarias españolas. Crue; Rebiun.

http://www.rebiun.org/documentos/Paginas/Jornadas-deAlfabetizaci\%C3\%B3n-Informacional-(ALFIN).aspx

Rebiun (2012). Competencias informáticas e informacionales en los estudios de grado. Edición revisada y ampliada. Comisión mixta CRUE-TIC y Rebiun.

http://www.rebiun.org/competenciadigital/Paginas/ documentacion.aspx

Revista española de documentación científica (2012). Competencias sin competencia: la alfin y sus circunstancias, $\mathrm{n}$. 35 , n. monográfico.

https://doi.org/10.3989/redc.2012.v35.imonografico

Secker, Jane; Coonan, Emma (2011). A new curriculum for information literacy: transitional, transferable, transformational. Curriculum and supporting documents. Arcadia Project; Cambridge University Library.

http://arcadiaproject.lib.cam.ac.uk/docs/ANCIL_final.pdf

Serra-Ferrer, Montserrat (2015). El comportament informacional en l'elaboració de treballs acadèmics: un estudi longitudinal als alumnes dels ensenyaments de ciències de la salut de la Fundació Universitària del Bages. Tesis doctoral. Universitat de Barcelona.

http://www.tdx.cat/handle/10803/315286

Sureda-Negre, Jaume; Comas-Forgas, Rubén (2006). Internet como fuente de documentación académica entre estudiantes universitarios. Palma de Mallorca: Fundación Universidad Empresa de las Islas Baleares. ISBN: 8469012703

Tumbleson, Beth E.; Burke, John J. (2010). "Embedded librarianship is job one: Building on instructional synergies". Public services quarterly, v. 6, n. 2/3, pp. 225-236.

https://doi.org/10.1080/15228959.2010.497457

Unión Europea (2006). "Recomendación del Parlamento Europeo y del Consejo, de 18 de diciembre de 2006 sobre las competencias clave para el aprendizaje permanente". Diario oficial de la Unión Europea, n. 394, 2006/962/CE, 30 de diciembre. https://www.boe.es/doue/2006/394/L00010-00018.pdf

Universidad de Alcalá (2007). "Modelo educativo de la Universidad de Alcalá". Boletín oficial de la Universidad de Alcalá. Avance, n. 6, junio.

https://portal.uah.es/portal/page/portal/secretaria_general/ boletin_oficial/2007/2007_Boletin_avance_junio.pdf

Universidad de Alcalá (2009). “Normativa reguladora de la oferta de asignaturas y actividades transversales en las titulaciones de grado". Boletín oficial de la Universidad de Alcalá, febrero.

https://portal.uah.es/portal/page/portal/secretaria_ general/boletin_oficial/2009/Febrero2009.pdf

Virkus, Sirje (2003), "Information literacy in Europe: a literature review". Information research, v. 8, n. 4. http://informationr.net/ir/8-4/paper159.html 